\title{
PROPERTIES OF POWERFUL SOLAR TYPE III BURSTS IN THE FREQUENCY RANGE OF 10-30 MHZ
}

\author{
A. I. Boiko*, V. N. Mel'nik*, A. A. Konovalenko*, H. O. Rucker ${ }^{\dagger}$, \\ E. P. Abranin*, V. V. Dorovskyy*, and A. Lecacheux ${ }^{\ddagger}$
}

\begin{abstract}
We report on the observation of powerful (fluxes are larger than $10^{-19} \mathrm{~W} / \mathrm{m}^{2} \mathrm{~Hz}$ ) solar type III bursts at frequencies of $10-30 \mathrm{MHz}$ using the radio telescope UTR 2. The properties of powerful type III bursts observed in July 2002 (163 bursts) and August 2002 (231 bursts) were analysed. It was determined that the majority of these bursts were observed on days when an active region was located near the central meridian or at longitudes of $40^{\circ}-60^{\circ}$ to the east or west of the central meridian. All of these bursts drift from high frequency to low with frequency drift rates of $1-2.5 \mathrm{MHz} / \mathrm{s}$ in most cases. We note that the frequency drift rate of these bursts increases linearly with frequency for all days of observation. This indicates that the solar corona above active regions shows an exponential density decrease with distance. The typical duration of the powerful type III bursts changes from 6 to $12 \mathrm{~s}$. The instantaneous frequency widths of these bursts do not depend on the day of observation. There is a linear dependence between the average frequency width and frequency. The implication of these observations are discussed in the frame of the plasma model for the type III bursts generation.
\end{abstract}

\section{Introduction}

Wild [Wild, 1950] first defined type III bursts as a individual type of solar radio burst. He obtained an inversely proportional dependence of duration versus frequency for 32 bursts in the frequency range of $70-130 \mathrm{MHz}$. He also found a linear approximation for the frequency drift rate via frequency dependence. More detailed analysis of type III bursts observed in the range of $50 \mathrm{kHz}-3.5 \mathrm{MHz}$ was made by Alvarez and Haddock [1973]. They found that the drift rate versus frequency relationship could be described by the

\footnotetext{
* Institute of Radio Astronomy, Ukrainian Academy of Sciences, Chervonopraporna 4, 61002, Kharkiv, Ukraine

† Space Research Institute, Austrian Academy of Sciences, Schmiedlstrasse 6, A-8042 Graz, Austria

‡LESIA, Observatoire de Paris, UMR CNRS 8109, 92195 Meudon, France
} 
equation $d f / d t \approx-0.01 f^{1.84}(d f / d t$ in $\mathrm{MHz} / \mathrm{s}, f$ in $\mathrm{MHz})$ and extended it to the band of $50 \mathrm{kHz}-1 \mathrm{GHz}$. Boischot [1967] analysed 75 type III bursts in the frequency range of $8-40 \mathrm{MHz}$. Their drift rates were $5-10 \mathrm{MHz} / \mathrm{s}$ at frequencies greater than $30 \mathrm{MHz}$ and $1-3 \mathrm{MHz} / \mathrm{s}$ at frequencies $10-15 \mathrm{MHz}$. At the same time the burst duration was about $10 \mathrm{~s}$ at $30 \mathrm{MHz}$. Hartz [1969] considered the observational properties of type III bursts observed in the frequency range of $0.1-15 \mathrm{MHz}$. The dependence of drift rate on frequency is similar to that of Alvarez and Haddock [1973]. Hartz extended this dependence up to $100 \mathrm{MHz}$. A more recent study of drift rates was presented by Mann et al. [1999]

The observational results of powerful (fluxes are larger than $10^{-19} \mathrm{~W} / \mathrm{m}^{2} \mathrm{~Hz}$ ) solar type III bursts in the decameter range $(10-30 \mathrm{MHz})$ during type III burst storms, which were registered in July and August 2002, are presented. The main characteristics of these bursts, such as frequency drift rates, durations, fluxes and instantaneous frequency bandwidths, are analysed. The properties of powerful type III bursts are discussed.

\section{Observations}

The powerful type III bursts discussed in the present paper were registered with the radio telescope UTR-2 (Kharkov, Ukraine) during the summer observational campaign of 2002. Three sections of the radio telescope with a total area of $30,000 \mathrm{~m}^{2}$ were used (Figure 1). It provides a beam of $1^{0} \times 13^{0}$. Data were acquired using the 60 -channel receiver with a frequency resolution of $300 \mathrm{kHz}$ and a time resolution of $10 \mathrm{~ms}$, and with a DSP (Digital Spectral Polarimeter) [Kleewein, 1997] with a frequency resolution of $12 \mathrm{kHz}$, a time resolution of $100 \mathrm{~ms}$ and a sensitivity of $100 \mathrm{Jy}$.

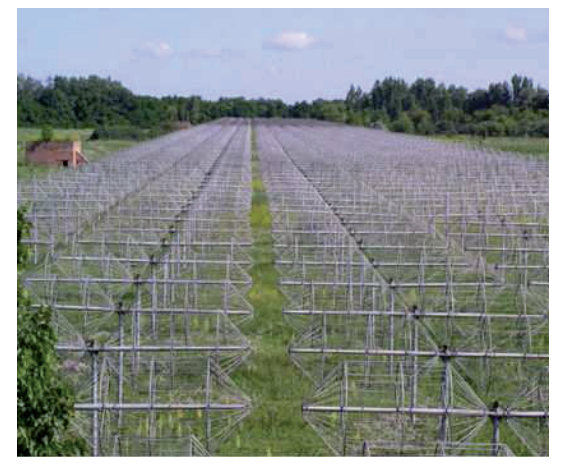

Figure 1: The radio telescope UTR-2, Kharkov, Ukraine

Data obtained for the powerful solar type III bursts (163 bursts in July 2002 and 231 bursts in August 2002) were processed. In the range of $10-30 \mathrm{MHz}$ all of these bursts have negative frequency drift rates (Figure 2a). The majority of powerful type III burst time profiles are not symmetrical (Figure $2 \mathrm{~b}$ ). 


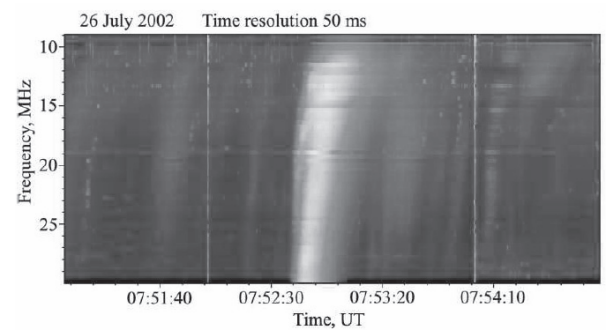

a)

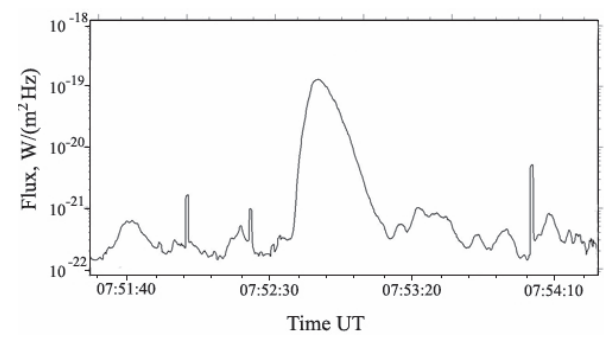

b)

Figure 2: A powerful type III burst (07:52:50 UT on 26 July 2002) against a background of type III burst storm (a) and its time profile at the frequency $26.15 \mathrm{MHz}$ (b).

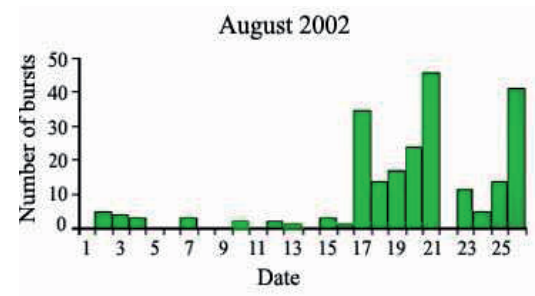

a)

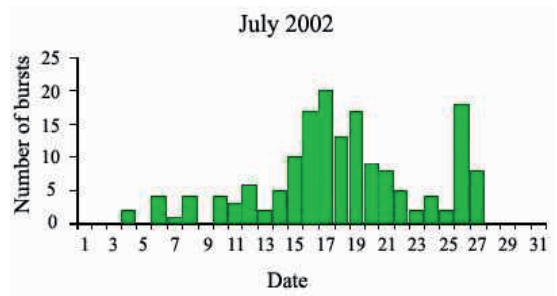

b)

Figure 3: The distribution of the powerful type III bursts

Most of the powerful type III bursts are observed on days when their occurrence is associated with active regions located near the central meridian or in the longitudes range $\pm 40^{\circ}-60^{\circ}$. The two maxima (on 17 and 26 of August) seen in the histogram (Figure $3 \mathrm{a}$ ) correspond to the intersection of the central meridian with two active regions. The maximum on the 21 of August is associated with 2 active regions not located in the center on the solar disc $\left(60^{\circ}\right.$ to the East and $50^{\circ}$ to the West from the central meridian). A similar situation occurs during the July 2002 storms (Figure 3b).

In most cases powerful type III bursts have frequency drift rates $(d f / d t)$ in the range of $1-3 \mathrm{MHz} / \mathrm{s}$. The drift rates versus frequency dependence for these bursts is shown 


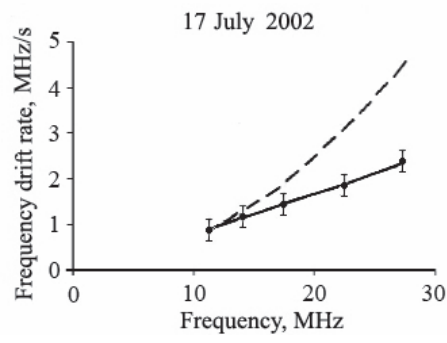

a)

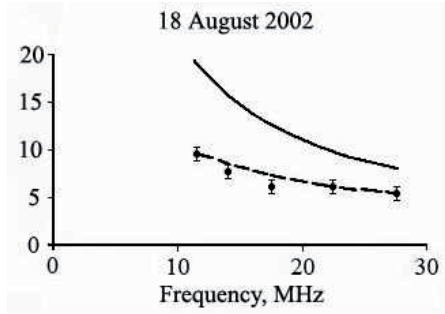

b)

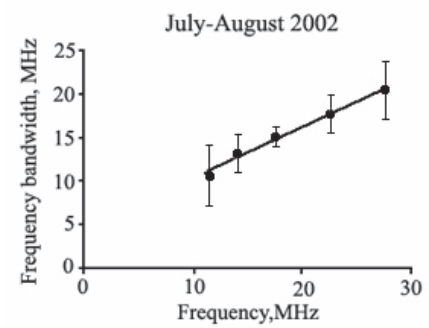

c)

Figure 4: Frequency drift rate versus frequency: dots represent observational values, dashed curve represents empirical Alvarez and Haddock's dependence [Alvarez and Haddock, 1973]) (a); duration versus frequency: squares represent observational values, solid curve represents the dependence in [Suzuki and Dulk, 1985], dashed curve represents the dependence in [Elgaroy and Lyngstad, 1972]) (b); average bandwidth versus frequency (c). 
in Figure 4a. We draw attention to the difference between the obtained and generally accepted empirical [Alvarez and Haddock, 1973] dependencies. Drift rates were almost the same at frequencies $10-15 \mathrm{MHz}$, but they differ considerably by a factor of about 2 at frequencies $20-30 \mathrm{MHz}$ and also depend on the location of an active region on the solar disc. We propose a linear approximation between drift rate and frequency

$$
d f / d t=-A \cdot f
$$

where $A$ is a coefficient depending on the day and on the storm. The coefficient $A$ is in the range of $0.08-0.09 \mathrm{~s}^{-1}$. We pointed out that connection between drift rate and frequency for powerful type III bursts for every day of observation is linear. Using the obtained linear equation, and supposing that type III radio emission is fundamental, $A$ is a constant value and the source velocity $v_{s}$ is also constant, the density dependence on distance $r$ can be found as follows

$$
n=n_{0} \cdot \exp \left(-2 \cdot A \cdot r / v_{s}\right),
$$

where $n_{0}$ is the normalization factor.

According to our observations the duration of these powerful type III bursts is in the range of $6-12$ seconds. The derived duration-frequency dependence (Figure $4 \mathrm{~b}$ ) is not so steep in comparison with the generally accepted equation $T=220 / f$ ( $T$ in s, $f$ in $\mathrm{MHz}$ ) [Suzuki and Dulk, 1985], but is in better agreed with the dependence $T=60 \cdot f^{-2 / 3}$ [Elgaroy and Lyngstad, 1972].

One of the important physical characteristics of powerful type III bursts is their instantaneous frequency bandwidth $\Delta f$. The dependence of the average frequency bandwidth on frequency (Figure 4c) is linear $\Delta f=0.6 \cdot f+3$ (frequency in $\mathrm{MHz}$ ). We consider that it is governed with the width of spectrum of the Langmuir waves generated by fast electrons. This width is equal to $\Delta k_{l} \approx 0.7 \cdot \omega_{p e} / v_{T e}$ and $\Delta k_{l} \approx 1.5 \cdot \omega_{p e} / c$ at fundamental and harmonic emission correspondingly [Melnik et al., 2010].

In all days of observation the powerful type III bursts flux dependence on frequency behaves in different ways: decrease with frequency, increase with frequency, a maximum value of flux is achieved in the range of $15-25 \mathrm{MHz}$.

\section{Conclusion}

A main result of our analysis of powerful type III bursts observed in the decameter range is the revelation of a linear dependence between their frequency drift rate and frequency in the range of $10-30 \mathrm{MHz}$. This result is repeated day after day during all observations. It indicates that the solar corona above active regions shows an exponential density decrease with distance. The study also proves that the durations of these bursts are a little smaller than usually accepted, but correspond to Elgaroy and Lyngstad's dependence. We confirm that both drift rates and durations depend on the position of an active region on the solar disc. 


\section{References}

Alvarez, H., and F. T. Haddock, Solar wind density model from km-wave type III bursts, Solar Phys., 29,197-209, 1973.

Boischot, A., Quelques caracteristiques des sursauts solaires de type III sur tre'ś basses freq̀uences, Annls Astrophys., 30, 1-7, 1967.

Elgaroy, O., and E. Lyngstad, High-resolution observations of Type III solar radio bursts, Astron. Astrophys., 16, 1-12, 1972.

Hartz, T. R., Type III solar radio noise bursts at hectometer wavelengths, Planet. Space Sci., 17, 267-287, 1969.

Kleewein, P., C. Rosolen, and A. Lecacheux, New digital spectrometers for ground based decameter radio astronomy, in Planetary Radio Emission IV, edited by H. O. Rucker, S. J. Bauer, and A. Lecacheux, Austrian Academy of Sciences Press, Vienna, 349 358, 1997.

Mann, G., F. Jansen, R. J. MacDowall, M. L. Kaiser, and R. G. Stone, A heliospheric density model and type III radio bursts, Astron. Astrophys., 348, 614-620, 1999.

Melnik, V. N., A. A. Konovalenko, H. O. Rucker, A. I. Boiko, V. V. Dorovskyy, E. P. Abranin, and A. Lecacheux, Observations of powerful type III bursts in the frequency range of 10-30 MHz, Solar Phys., 269, 2, 335-350, 2011.

Suzuki, S., and G. A. Dulk, Bursts of Type III and Type V, in Solar Physics, edited by D. J. McLean, and N. R. Labrum, 285, Cambridge Univ. Press, Cambridge, 289-332, 1985.

Wild, J. P., Observations of the spectrum of high-intensity Solar radiation at meter wavelengths - III. Isolated bursts, Austr. J. Sci. Res., 3, 541-557, 1950. 